DOI: http://dx.doi.org/10.21123/bsj.2016.13.2.0394

\title{
On the Representations of M-Groups
}

Taghreed Hur Majeed*

\section{Khawla A.Al-Zubaidy*** \\ Niran Sabah Jasim***}

* Department of Mathematics, College of Education, Al-Mustansiriya University
** Department of Mechanic Engineering, College of Engineering, University of
Baghdad
***Department of Mathematics, College of Education for Pure Science \Ibn-Al-
Haitham, University of Baghdad

Received 26/3/ 2015

Accepted 3/6/ 2015

\section{(1) $\odot \odot$}

EY NG NDThis work is licensed under a Creative Commons Attribution-

NonCommercial-NoDerivatives 4.0 International Licens

\begin{abstract}
:
The main object of this paper is to study the representations of monomial groups and characters technique for representations of monomial groups. We refer to monomial groups by M-groups. Moreover we investigate the relation of monomial groups and solvable groups.

Many applications have been given the symbol G e.g. group of order 297 is an M-group and solvable. For any group $G$, the factor group $G / G^{\prime}\left(G^{\prime}\right.$ is the derived subgroup of $G)$ is an M-group in particular if $G=S_{n}, S L(4, R)$.
\end{abstract}

Key words: Representation Theory, Monomial Group, Solvable Group, Representation of Monomial Group.

\section{Introduction:}

The theory of groups really begins with Galois (1811-1923) who demonstrates that polynomials are best understood by examining certain groups of permutations of their roots. Since that time, groups have arisen in almost every branch of mathematics. [1] The essential body of representation theory has been constructed by Richard Brauer (19011977). His processors; Frobenius Burnside and Schur, give the grand task to which character theory could make a central contribution, that is, the complete classification of finite simple groups [2].

In studying monomial group it is important to know as much as possible about the character of its subgroups. A finite group is called monomial group (M-group) if every irreducible complex character is induced from a linear character of a subgroup of G. No nonlinear character theoretic description of the class of M-groups has been found.

The process of imbedding a group in a larger group of some prescribed type has been one of the most useful tools in the investigation of properties of groups. The three principal types of representation of groups, each with its particular field of usefulness, are the following:

1. Permutation groups.

2. Monomial groups. 
3. Linear or matrix representations of groups.

These three types of representation correspond to an imbedding of the group in the following groups:

1 . The symmetric group.

2. The complete monomial group.

3. The full linear group, [3]

A finite group is termed monomial (or sometimes, a M-group or $\mathbf{M}_{1}$-group) with respect to a field $\mathrm{K}$ (whose characteristic does not divide the group order) if it satisfies the following equivalent conditions:

1. Every irreducible representation of the group over $\mathrm{K}$ is induced from a one-dimensional representation of a subgroup, i.e., a linear character.

2. Every finite-dimensional linear representation of the group over $\mathrm{K}$ is a monomial linear representation: it is a direct sum of representations induced from onedimensional representations of subgroups, [4].

Recently, Lakshmibai 2011 [5], Musili 2011 [6] and Seshadri 2012 [7] give detailed descriptions of the early development of standard monomial theory.

Bravi, Chirivi, Gendini and Maffei 2015 [8] study the standard monomial theory for wonderful varieties.

Hajim in 2007 [3] gave new result on representation of monomial groups and character technique for representation of monomial group.

Here we give more results on representation of monomial groups and discuss more properties of monomial groups and solvable groups.

\section{Representation and Solvable Groups:}

The main aim of this section is to introduce the most basic definitions and facts in ordinary representation theory and solvable group.

\section{Definition 1: [2]}

A group homomorphism $\pi: \mathrm{G} \longrightarrow$ $\mathrm{GL}(\mathrm{V})$ where $\mathrm{G}$ is a group and $\mathrm{V}$ is a vector space which is called a representation of $\mathrm{G}$ in $\mathrm{V}$.

\section{Proposition 2: [2]}

There is a bijective correspondence between the set of linear actions of $G$ on vector space $\mathrm{V}$ and the set of homomorphisms from G to GL(V).

\section{Example 3:}

Consider the cyclic group $\mathrm{C}_{5}=$ $\left\{1, \mathrm{x}, \mathrm{x}^{2}, \mathrm{x}^{3}, \mathrm{x}^{4}\right\}$. And let $\pi: \mathrm{C}_{5} \longrightarrow$ GL(V), the $5^{\text {th }}$ root of unity $\left\{1, \mathrm{x}, \mathrm{x}^{2}, \mathrm{x}^{3}, \mathrm{x}^{4}\right\}$ are:

$\pi_{1}(\mathrm{x})=1$

$\pi_{2}(\mathrm{x})=\mathrm{e}^{2 \pi \mathrm{i} / 5}$

$\pi_{3}\left(\mathrm{x}^{2}\right)=\left(\mathrm{e}^{2 \pi \mathrm{i} / 5}\right)^{2}$

$\pi_{4}\left(\mathrm{x}^{3}\right)=\left(\mathrm{e}^{2 \pi \mathrm{i} / 5}\right)^{3}$

$\pi_{5}\left(x^{4}\right)=\left(e^{2 \pi i / 5}\right)^{4}$.

Definition4: [9]

A group $G$ is said to be a solvable group, if there exists a finite chain of subgroups $\mathrm{G}=\mathrm{H}_{0} \supseteq \mathrm{H}_{1} \supseteq \mathrm{H}_{2} \supseteq \ldots \supseteq \mathrm{H}_{\mathrm{k}}$ $=\{\mathrm{e}\}$ such that $\mathrm{H}_{\mathrm{i}} \unlhd \mathrm{H}_{\mathrm{i}}-1$ and the quotient group $\mathrm{H}_{\mathrm{i}-1} / \mathrm{H}_{\mathrm{i}}$ is abelian, where $\unlhd$ is the symbol of normal subgroup.

Example 5: [1]

The symmetric group $\mathrm{S}_{4}$ is solvable.

\section{Definition 6: [1]}

A group $G$ is called nilpotent if it has a central series, that is a normal series $1=\mathrm{G}_{0} \subseteq \mathrm{G}_{1} \subseteq \ldots \subseteq \mathrm{G}_{\mathrm{n}}=\mathrm{G}$ such that $G_{i+1} / G_{i}$ is contained in the center of $G / G_{i}$ for all $i$ (a finite group all of whose subgroup are normal is nilpotent).

\section{Characters and M-Groups:}

Character theory was developed by Frobenius in 1896. It provides a powerful tool for proving theorems about finite groups. We use character 
techniques to obtain more information and facts about M-groups.

\section{Definition 1: [10]}

Let $\chi$ be a character of $\mathrm{G}$. Then $\chi$ is monomial if $\chi=\lambda^{\mathrm{G}}$ where $\lambda$ is a linear character of some subgroup of $\mathrm{G}$.

\section{Remark 2: [11]}

Let $G$ be any group, we denote by $\operatorname{Irr}(G)$ for the set of all irreducible character of $\mathrm{G}$.

\section{Definition 3: [11]}

A group $\mathrm{G}$ is an M-group (monomial group) if every $\chi \in \operatorname{Irr}(\mathrm{G})$ is monomial character.

Theorem 4: [1]

Every abelian group is a nilpotent group.

\section{Theorem (Taketa) 5: [10]}

Every M-group is a solvable group.

\section{Theorem (Taketa) 6 : [9]} group.

Every nilpotent group is an $\mathrm{M}$ -

\section{Proposition 7: [2]}

A finite $\mathrm{M}$-group $\mathrm{G}$ of order $\mathrm{n}$ has exactly $\mathrm{n}$ distinct characters.

\section{Example 8:}

The Klein 4-group is given by $\mathrm{V}=$ $\langle\mathrm{x}\rangle \mathrm{x}\langle\mathrm{y}\rangle$ where $\mathrm{x}^{2}=\mathrm{y}^{2}=1, \mathrm{xy}=\mathrm{yx}$. In order to obtain the character of $\mathrm{V}$, we have to compute

$$
\begin{aligned}
& \chi_{[1,0]}((1,1))=\mathrm{e}^{2 \pi \mathrm{i}(1 \cdot 0+0 \cdot 0) / 2}=1 \\
& \chi_{[1,0]}((\mathrm{x}, 1))=\mathrm{e}^{2 \pi \mathrm{i}(1 \cdot 1+0 \cdot 0) / 2}=-1 \\
& \chi_{[1,0]}((1, \mathrm{y}))=\mathrm{e}^{2 \pi \mathrm{i}(1 \cdot 0+0 \cdot 1) / 2}=1 \\
& \chi_{[1,0]}((\mathrm{x}, \mathrm{y}))=\mathrm{e}^{2 \pi \mathrm{i}(1 \cdot 1+0 \cdot 1) / 2}=-1 \\
& \chi_{[0,1]}((1,1))=\mathrm{e}^{2 \pi \mathrm{i}(0 \cdot 0+1 \cdot 0) / 2}=1 \\
& \chi_{[0,1]}((\mathrm{x}, 1))=\mathrm{e}^{2 \pi \mathrm{i}(0 \cdot 1+1 \cdot 0) / 2}=1 \\
& \chi_{[0,1]}((1, \mathrm{y}))=\mathrm{e}^{2 \pi \mathrm{i}(0 \cdot 0+1 \cdot 1) / 2}=-1 \\
& \chi_{[0,1]}((\mathrm{x}, \mathrm{y}))=\mathrm{e}^{2 \pi \mathrm{i}(0 \cdot 1+1 \cdot 1) / 2}=-1 \\
& \chi_{[1,1]}((1,1))=\mathrm{e}^{2 \pi \mathrm{i}(1 \cdot 0+1 \cdot 0) / 2}=1 \\
& \chi_{[1,1]}((\mathrm{x}, 1))=\mathrm{e}^{2 \pi \mathrm{i}(1 \cdot 1+1 \cdot 0) / 2}=-1 \\
& \chi_{[1,1]}((1, \mathrm{y}))=\mathrm{e}^{2 \pi \mathrm{i}(1 \cdot 0+1 \cdot 1) / 2}=-1
\end{aligned}
$$

$\chi_{[1,1]}((\mathrm{x}, \mathrm{y}))=\mathrm{e}^{2 \pi \mathrm{i}(1 \cdot 1+1 \cdot 1) / 2}=1$

Table (1) shows the results:

Table (1) Character Table of $\mathbf{G}=$ $\langle\mathbf{x}>\mathrm{x}\langle\mathrm{y}\rangle$

\begin{tabular}{|c|c|c|c|c|}
\hline $\mathrm{C}_{\alpha}$ & $(1,1)$ & $(\mathrm{x}, 1)$ & $(1, \mathrm{y})$ & $(\mathrm{x}, \mathrm{y})$ \\
\hline$\left|\mathrm{C}_{\alpha}\right|$ & 1 & 1 & 1 & 1 \\
\hline$\left|\mathrm{C}_{\mathrm{G}}\left(\mathrm{C}_{\alpha}\right)\right|$ & 4 & 4 & 4 & 4 \\
\hline$\chi_{[0,0]}$ & 1 & 1 & 1 & 1 \\
\hline$\chi_{[1,0]}$ & 1 & -1 & 1 & -1 \\
\hline$\chi_{[0,1]}$ & 1 & 1 & -1 & -1 \\
\hline$\chi_{[1,1]}$ & 1 & -1 & -1 & 1 \\
\hline
\end{tabular}

\section{Main Results and Applications:}

Now, we give the main results and applications of monomial groups and solvable groups.

\section{Proposition 1: [9]}

If $(\mathrm{H}, *),(\mathrm{K}, *)$ are subgroups of the group $(\mathrm{G}, *)$ such that $\mathrm{H} * \mathrm{~K}=\mathrm{K} * \mathrm{H}$, then $(\mathrm{H} * \mathrm{~K}, *)$ is a subgroup of $(\mathrm{G}, *)$.

\section{Proposition 2:}

If $\left(\mathrm{H}_{1} * \mathrm{~K}_{1}, *\right)$ and $\left(\mathrm{H}_{2} * \mathrm{~K}_{2}, *\right)$ are two abelian subgroups of a group $(\mathrm{G}, *)$ then $\left(\mathrm{H}_{1} * \mathrm{~K}_{1}\right) \times\left(\mathrm{H}_{2} * \mathrm{~K}_{2}\right)$ and $\left(\mathrm{H}_{2} * \mathrm{~K}_{2}\right) \times\left(\mathrm{H}_{1} * \mathrm{~K}_{1}\right)$ are isomorphic and $\left(\mathrm{H}_{1} * \mathrm{~K}_{1}\right) \times\left(\mathrm{H}_{2} * \mathrm{~K}_{2}\right)$ is $\mathrm{M}$-group hence it is solvable group.

Proof:

Since $\left(\mathrm{H}_{1} * \mathrm{~K}_{1}, *\right)$ and $\left(\mathrm{H}_{2} * \mathrm{~K}_{2}, *\right)$ are abelian groups, then $\left(\mathrm{H}_{1} * \mathrm{~K}_{1}\right) \times\left(\mathrm{H}_{2} * \mathrm{~K}_{2}\right)$ and $\left(\mathrm{H}_{2} * \mathrm{~K}_{2}\right) \times\left(\mathrm{H}_{1} * \mathrm{~K}_{1}\right)$ are abelian groups, so $\left(\mathrm{H}_{1} * \mathrm{~K}_{1}\right) \times\left(\mathrm{H}_{2} * \mathrm{~K}_{2}\right)$ and $\left(\mathrm{H}_{2} * \mathrm{~K}_{2}\right) \times\left(\mathrm{H}_{1} * \mathrm{~K}_{1}\right)$ are nilpotent by Theorem (3.4), therefore $\left(\mathrm{H}_{1} * \mathrm{~K}_{1}\right) \times\left(\mathrm{H}_{2} * \mathrm{~K}_{2}\right)$ and $\left(\mathrm{H}_{2} * \mathrm{~K}_{2}\right) \times\left(\mathrm{H}_{1} * \mathrm{~K}_{1}\right)$ are $\mathrm{M}$-group by Theorem (3.6). Hence $\left(\mathrm{H}_{1} * \mathrm{~K}_{1}\right) \times\left(\mathrm{H}_{2} * \mathrm{~K}_{2}\right)$ and $\left(\mathrm{H}_{2} * \mathrm{~K}_{2}\right) \times\left(\mathrm{H}_{1} * \mathrm{~K}_{1}\right)$ are solvable group by Theorem (5).

Proposition 3: [9]

Let $\mathrm{H}_{1}, \mathrm{H}_{2}, \ldots, \mathrm{H}_{\mathrm{n}}-{ }_{1}, \mathrm{H}_{\mathrm{n}}$ be a subgroups of the group $\mathrm{G}$, then $\mathrm{H}_{1} * \mathrm{H}_{2} * \ldots * \mathrm{H}_{\mathrm{n}-1} * \mathrm{H}_{\mathrm{n}}$ and $\mathrm{H}_{\mathrm{n}} * \mathrm{H}_{\mathrm{n}-1} *$ $\mathrm{H}_{2} * \quad \mathrm{H}_{1}$ are isomorphic and $\mathrm{H}_{1} * \mathrm{H}_{2} * \ldots * \mathrm{H}_{\mathrm{n}-1} * \mathrm{H}_{\mathrm{n}}$ is abelian only if $\mathrm{H}_{1}, \mathrm{H}_{2}, \ldots, \mathrm{H}_{\mathrm{n}-1}, \mathrm{H}_{\mathrm{n}}$ are abelian. 
Proof:

It is a simple substantiation that $\mathrm{H}_{1} * \mathrm{H}_{2} * \ldots * \mathrm{H}_{\mathrm{n}}-1 * \mathrm{H}_{\mathrm{n}}$ and $\mathrm{H}_{\mathrm{n}} * \mathrm{H}_{\mathrm{n}}$ $1 * \mathrm{H}_{2} * \mathrm{H}_{1}$ are isomorphic under the mapping $\left(\mathrm{h}_{1}, \mathrm{~h}_{2} \ldots, \mathrm{h}_{\mathrm{n}-1}, \mathrm{~h}_{\mathrm{n}}\right) \longrightarrow\left(\mathrm{h}_{\mathrm{n}}, \mathrm{h}_{\mathrm{n}-}\right.$ $\left.{ }_{1}, \mathrm{~h}_{2}, \mathrm{~h}_{1}\right)$ where $\quad \mathrm{h}_{1} \in \mathrm{H}_{1}, \mathrm{~h}_{2} \in$ $\mathrm{H}_{2}, \ldots, \mathrm{h}_{\mathrm{n}-1} \in \mathrm{H}_{\mathrm{n}-1}, \mathrm{~h}_{\mathrm{n}} \in \mathrm{H}_{\mathrm{n}}$. Let $\left(h_{1}, h_{2}, \ldots, h_{n-1}, h_{n}\right),\left(h_{1}^{\prime}, h_{2}^{\prime}, \ldots, h_{n-1}^{\prime}, h_{n}^{\prime}\right)$ be elements of $\mathrm{H}_{1} * \mathrm{H}_{2} * \ldots * \mathrm{H}_{\mathrm{n}}-{ }_{1} * \mathrm{H}_{\mathrm{n}}$. Then

$\left(\mathrm{h}_{1}, \mathrm{~h}_{2}, \ldots, \mathrm{h}_{\mathrm{n}-1}, \mathrm{~h}_{\mathrm{n}}\right) *\left(\mathrm{~h}_{1}^{\prime}, \mathrm{h}_{2}^{\prime}, \ldots, \mathrm{h}_{\mathrm{n}-1}^{\prime}, \mathrm{h}_{\mathrm{n}}^{\prime}\right)=$ $\left(\left(h_{1} * h_{1}^{\prime}\right) *\left(h_{2} * h_{2}^{\prime}\right), \ldots,\left(h_{n-1} * h_{n-1}^{\prime}\right),\left(h_{n} * h_{n}^{\prime}\right)\right)$ if and only if $h_{1} * h_{1}^{\prime}=h_{1}^{\prime} * h_{1}, h_{2} * h_{2}^{\prime}=h_{2}^{\prime} * h_{2}, \ldots, h_{n-1} * h_{n-1}^{\prime}=h_{n-1}^{\prime} * h_{n-1}$ and $\mathrm{h}_{\mathrm{n}} * \mathrm{~h}_{\mathrm{n}}^{\prime}=\mathrm{h}_{\mathrm{n}}^{\prime} * \mathrm{~h}_{\mathrm{n}}$ for all $\mathrm{h}_{\mathrm{i}}, \mathrm{h}_{\mathrm{i}}^{\prime}$ $\in \mathrm{H}_{\mathrm{i}}, \mathrm{i}=1,2, \ldots, \mathrm{n}$. So $\mathrm{H}_{1} * \mathrm{H}_{2} * \ldots * \mathrm{H}_{\mathrm{n}-}$ $1 * \mathrm{H}_{\mathrm{n}}$ is abelian if and only if $\mathrm{H}_{1}, \mathrm{H}_{2}$, $\ldots, \mathrm{H}_{\mathrm{n}-1}, \mathrm{H}_{\mathrm{n}}$ are abelian.

\section{Example 4:}

Let $\mathrm{G}$ be group of order 297 then it is M-group and solvable group where $297=3^{3} \times 11, \mathrm{G}$ has a 3 -sylow subgroup H. It is of order 27, and a 11-sylow subgroup K of order 11.

Let $\mathrm{H}$ have $\mathrm{n}$ conjugates. Then $\mathrm{n}=1+$ $3 r(r \geq 0)$ and divides 297 and so we conclude easily that as the factors of 297 are $1,3,9,11,27,33,99,297, \mathrm{n}=1$ and hence $\mathrm{H}$ is normal in $\mathrm{G}$.

Similarly, $\mathrm{K}$ is normal in $\mathrm{G}$, we have $\mathrm{G}$ $=\mathrm{HK}$. Since $|\mathrm{HK}|=|\mathrm{HK}| /|\mathrm{H} \cap \mathrm{K}|=$
$|\mathrm{H}||\mathrm{K}|=297$, thus $\mathrm{G}$ is isomorphic to the direct product $\mathrm{H} * \mathrm{~K}$. But $\mathrm{H}$ is abelian and $\mathrm{K}$ is cyclic and thus by Proposition (3) HK is an abelian subgroup. Hence G is abelian then $G$ is nilpotent by Theorem (4). Now G is M-group by Theorem (6).

\section{Proposition 5: [9]}

Let $\mathrm{H}_{1}, \mathrm{H}_{2}, \ldots, \mathrm{H}_{\mathrm{n}}$ and $\mathrm{K}_{1}, \mathrm{~K}_{2}, \ldots, \mathrm{K}_{\mathrm{n}}$ be subgroups of the group $\mathrm{G}$, then $\left(\mathrm{H}_{1} * \mathrm{~K}_{1}\right) \times\left(\mathrm{H}_{2} * \mathrm{~K}_{2}\right) \times \ldots \times\left(\mathrm{H}_{\mathrm{n}} * \mathrm{~K}_{\mathrm{n}}\right) \quad$ and $\left(\mathrm{H}_{\mathrm{n}} * \mathrm{~K}_{\mathrm{n}}\right) \times \ldots \times\left(\mathrm{H}_{2} * \mathrm{~K}_{2}\right) \times\left(\mathrm{H}_{1} * \mathrm{~K}_{1}\right) \quad$ are isomorphic and $\left(\mathrm{H}_{1} * \mathrm{~K}_{1}\right) \times\left(\mathrm{H}_{2} * \mathrm{~K}_{2}\right) \times \ldots \times\left(\mathrm{H}_{\mathrm{n}} * \mathrm{~K}_{\mathrm{n}}\right) \quad$ is abelian if \& only if $\left(\mathrm{H}_{1} * \mathrm{~K}_{1}\right),\left(\mathrm{H}_{2} * \mathrm{~K}_{2}\right)$, $\ldots,\left(\mathrm{H}_{\mathrm{n}} * \mathrm{~K}_{\mathrm{n}}\right)$ are abelian.

Proof:

It is an easy confirmation that $\left(\mathrm{H}_{1} * \mathrm{~K}_{1}\right) \times\left(\mathrm{H}_{2} * \mathrm{~K}_{2}\right) \times \ldots \times\left(\mathrm{H}_{\mathrm{n}} * \mathrm{~K}_{\mathrm{n}}\right) \quad$ and $\left(\mathrm{H}_{\mathrm{n}} * \mathrm{~K}_{\mathrm{n}}\right) \times \ldots \times\left(\mathrm{H}_{2} * \mathrm{~K}_{2}\right) \times\left(\mathrm{H}_{1} * \mathrm{~K}_{1}\right) \quad$ are isomorphic under the mapping

$$
\left(\mathrm{h}_{1} * \mathrm{k}_{1}, \quad \mathrm{~h}_{2} * \mathrm{k}_{2}, \ldots, \mathrm{h}_{\mathrm{n}} * \mathrm{k}_{\mathrm{n}}\right)
$$

$\left(\mathrm{h}_{\mathrm{n}} * \mathrm{k}_{\mathrm{n}}, \ldots, \mathrm{h}_{2} * \mathrm{k}_{2}, \mathrm{~h}_{1} * \mathrm{k}_{1}\right)$ where $\mathrm{h}_{\mathrm{i}} \in \mathrm{H}_{\mathrm{i}}, \mathrm{k}_{\mathrm{i}}$ $\in \mathrm{K}_{\mathrm{i}}, \quad \mathrm{i}=1,2, \ldots, \mathrm{n}$ Allow $\left(\mathrm{h}_{1} * \mathrm{k}_{1}, \mathrm{~h}_{2} * \mathrm{k}_{2}, \ldots, \mathrm{h}_{\mathrm{n}} * \mathrm{k}_{\mathrm{n}}\right)$,

$$
\left(\mathrm{h}_{1}^{\prime} * \mathrm{k}_{1}^{\prime}, \mathrm{h}_{2}^{\prime} * \mathrm{k}_{2}^{\prime}, \ldots, \mathrm{h}_{\mathrm{n}}^{\prime} * \mathrm{k}_{\mathrm{n}}^{\prime}\right) \quad \text { be }
$$

elements of

$\left(\mathrm{H}_{1} * \mathrm{~K}_{1}\right) \times\left(\mathrm{H}_{2} * \mathrm{~K}_{2}\right) \times \ldots \times\left(\mathrm{H}_{\mathrm{n}} * \mathrm{~K}_{\mathrm{n}}\right)$. Thus

$\left(\mathrm{h}_{1} * \mathrm{k}_{1}, \mathrm{~h}_{2} * \mathrm{k}_{2}, \ldots, \mathrm{h}_{\mathrm{n}} * \mathrm{k}_{\mathrm{n}}\right)$

$\left(\mathrm{h}_{1}^{\prime} * \mathrm{k}_{1}^{\prime}, \mathrm{h}_{2}^{\prime} * \mathrm{k}_{2}^{\prime}, \ldots, \mathrm{h}_{\mathrm{n}}^{\prime} * \mathrm{k}_{\mathrm{n}}^{\prime}\right)=$

$$
\left(\left(\mathrm{h}_{1} * \mathrm{k}_{1}\right)\left(\mathrm{h}_{1}^{\prime} * \mathrm{k}_{1}^{\prime}\right),\left(\mathrm{h}_{2} * \mathrm{k}_{2}\right)\left(\mathrm{h}_{2}^{\prime} * \mathrm{k}_{2}^{\prime}\right), \ldots,\left(\mathrm{h}_{\mathrm{n}} * \mathrm{k}_{\mathrm{n}}\right)\left(\mathrm{h}_{\mathrm{n}}^{\prime} * \mathrm{k}_{\mathrm{n}}^{\prime}\right)\right)
$$

if and only if

$$
\left(\mathrm{h}_{1} * \mathrm{k}_{1}\right)\left(\mathrm{h}_{1}^{\prime} * \mathrm{k}_{1}^{\prime}\right)=\left(\mathrm{h}_{1}^{\prime} * \mathrm{k}_{1}^{\prime}\right)\left(\mathrm{h}_{1} * \mathrm{k}_{1}\right),\left(\mathrm{h}_{2} * \mathrm{k}_{2}\right)\left(\mathrm{h}_{2}^{\prime} * \mathrm{k}_{2}^{\prime}\right)=\left(\mathrm{h}_{2}^{\prime} * \mathrm{k}_{2}^{\prime}\right)\left(\mathrm{h}_{2} * \mathrm{k}_{2}\right)
$$

$\&\left(\mathrm{~h}_{\mathrm{n}} * \mathrm{k}_{\mathrm{n}}\right)\left(\mathrm{h}_{\mathrm{n}}^{\prime} * \mathrm{k}_{\mathrm{n}}^{\prime}\right)=\left(\mathrm{h}_{\mathrm{n}}^{\prime} * \mathrm{k}_{\mathrm{n}}^{\prime}\right)\left(\mathrm{h}_{\mathrm{n}} * \mathrm{k}_{\mathrm{n}}\right)$

for all $\mathrm{h}_{\mathrm{i}} * \mathrm{k}_{\mathrm{i}}, \mathrm{h}_{\mathrm{i}}^{\prime} * \mathrm{k}_{\mathrm{i}}^{\prime} \in \mathrm{H}_{\mathrm{i}} * \mathrm{~K}_{\mathrm{i}}, \mathrm{i}=$ $1,2, \ldots, \mathrm{n}$.

$\left(\mathrm{H}_{1} * \mathrm{~K}_{1}\right) \times\left(\mathrm{H}_{2} * \mathrm{~K}_{2}\right) \times \ldots \times\left(\mathrm{H}_{\mathrm{n}} * \mathrm{~K}_{\mathrm{n}}\right)$ abelian if and only if $\left(\mathrm{H}_{1} * \mathrm{~K}_{1}\right) \times\left(\mathrm{H}_{2} * \mathrm{~K}_{2}\right) \times \ldots \times\left(\mathrm{H}_{\mathrm{n}} * \mathrm{~K}_{\mathrm{n}}\right) \quad$ are abelian.

\section{Proposition 6:}

If $\mathrm{G}=\left\langle\mathrm{Z}_{1}\right\rangle \times\left\langle\mathrm{Z}_{2}\right\rangle \times \ldots \times\left\langle\mathrm{Z}_{\mathrm{m}}\right\rangle$ is an abelian group where $Z_{i}$ is a group of order $n_{i}$ and $|G|=\begin{array}{lllll}n_{1} & n_{2} & \ldots & n_{i} \text {, }\end{array}$ $\mathrm{X}=\mathrm{Z}_{1}^{\mathrm{a}_{1}} \mathrm{Z}_{2}^{\mathrm{a}_{2}} \ldots \mathrm{Z}_{\mathrm{m}}^{\mathrm{a}_{\mathrm{m}}}, 0 \leq \mathrm{a}_{\mathrm{i}} \leq \mathrm{n}_{\mathrm{i}}, \mathrm{i}=$

$1,2, \ldots, m$ then $\chi_{[r]}(x)=e^{2 \pi i \sum_{i=1}^{m} a_{i} r_{i} / n_{i}}$ is a monomial character and $\mathrm{G}$ is M-group. 
Proof:

$\mathrm{G}=\left\langle\mathrm{Z}_{1}\right\rangle \times\left\langle\mathrm{Z}_{2}\right\rangle \times \ldots \times\left\langle\mathrm{Z}_{\mathrm{m}}\right\rangle$ is an abelian group thus $\left\langle\mathrm{Z}_{1}\right\rangle,\left\langle\mathrm{Z}_{2}\right\rangle, \ldots$, $\left\langle\mathrm{Z}_{\mathrm{m}}\right\rangle$ are abelian since $\mathrm{H} \times \mathrm{K}$ is an abelian group iff $\mathrm{H}, \mathrm{K}$ are abelian group and $\left\langle\mathrm{Z}_{1}\right\rangle \times\left\langle\mathrm{Z}_{2}\right\rangle \times \ldots \times$ $\left\langle\mathrm{Z}_{\mathrm{m}}\right\rangle$ isomorphic to $\left\langle\mathrm{Z}_{\mathrm{m}}\right\rangle \times \ldots \times\left\langle\mathrm{Z}_{2}\right\rangle$ $\left.x<Z_{1}\right\rangle$. Every abelian group is M-group and the sum of character is character $\chi_{i}$ $\in \operatorname{Irr}(\mathrm{G})$ (irreducible of $\mathrm{G}), \mathrm{i}=1,2, \ldots, \mathrm{m}$ thus write $\chi_{1}=\lambda_{1}^{\mathrm{G}}$ and $\chi_{2}=\lambda_{2}^{\mathrm{G}}, \ldots$, $\chi_{\mathrm{m}}=\lambda_{\mathrm{m}}^{\mathrm{G}}$ where $\lambda_{1} \in \operatorname{Irr}\left(\mathrm{U}_{1}\right)$ and $\lambda_{2} \in$ $\operatorname{Irr}\left(\mathrm{U}_{2}\right), \ldots, \lambda_{\mathrm{m}} \in \operatorname{Irr}\left(\mathrm{U}_{\mathrm{m}}\right)$ are linear characters. Let $\mathrm{v} \in \operatorname{irr}\left(\mathrm{U}_{1} \cap \mathrm{U}_{2} \cap \ldots \cap \mathrm{U}_{\mathrm{m}}\right)$ be defined by $\mathrm{v}=\lambda_{1_{\left(\mathrm{U}_{1} \cap \mathrm{U}_{2} \cap \ldots \cap \mathrm{U}_{\mathrm{m}}\right)}} \lambda_{2_{\left(\mathrm{U}_{1} \cap \mathrm{U}_{2} \cap \ldots \cap \mathrm{U}_{\mathrm{m}}\right)}} \ldots \lambda_{\mathrm{m}_{\left(\mathrm{U}_{1} \cap \mathrm{U}_{2} \cap \ldots \cap \mathrm{U}_{\mathrm{m}}\right.}}$ and note that $\mathrm{v}$ is consistuent of $\chi_{\left(\mathrm{U}_{1} \cap \mathrm{U}_{2} \cap \ldots \cap \mathrm{U}_{\mathrm{m}}\right)}$, so that $\chi$ is a consistuent of $v^{\mathrm{G}}$. We have however

$\mathrm{v}^{\mathrm{G}}(1)=\left|\mathrm{G}: \mathrm{U}_{1} \cap \mathrm{U}_{2} \cap \ldots \cap \mathrm{U}_{\mathrm{m}}\right| \leq\left|\mathrm{G}: \mathrm{U}_{1}\right| \mid \mathrm{G}:$ $\mathrm{U}_{2}|\ldots| \mathrm{G}: \mathrm{U}_{\mathrm{m}} \mid=\chi_{1}(1) \chi_{21}(1) \ldots \chi_{\mathrm{m}}(1)=\chi(1)$ and thus $\chi=\mathrm{v}^{\mathrm{G}}$.

\section{Lemma 7:}

If $\mathrm{H}_{\mathrm{i}}, \mathrm{K}_{\mathrm{i}}, \mathrm{i}=1,2, \ldots, \mathrm{n}$ are two subgroups of the group $\mathrm{G}$ and $\left(\mathrm{H}_{1} * \mathrm{~K}_{1}\right)$, $\left(\mathrm{H}_{2} * \mathrm{~K}_{2}\right), \quad \ldots, \quad\left(\mathrm{H}_{\mathrm{n}} * \mathrm{~K}_{\mathrm{n}}\right)$ are abelian subgroups, then $\left(\mathrm{H}_{1} * \mathrm{~K}_{1}\right) \times\left(\mathrm{H}_{2} * \mathrm{~K}_{2}\right) \times \ldots \times\left(\mathrm{H}_{\mathrm{n}} * \mathrm{~K}_{\mathrm{n}}\right)$ is $\mathrm{M}$ group, hence a solvable group.

\section{Proof:}

Since $\left(\mathrm{H}_{1} * \mathrm{~K}_{1}\right),\left(\mathrm{H}_{2} * \mathrm{~K}_{2}\right), \ldots,\left(\mathrm{H}_{\mathrm{n}} * \mathrm{~K}_{\mathrm{n}}\right)$ are abelian subgroups, thus $\left(\mathrm{H}_{1} * \mathrm{~K}_{1}\right) \times\left(\mathrm{H}_{2} * \mathrm{~K}_{2}\right) \times \ldots \times\left(\mathrm{H}_{\mathrm{n}} * \mathrm{~K}_{\mathrm{n}}\right)$ is an abelian group by Proposition (3). So $\left(\mathrm{H}_{1} * \mathrm{~K}_{1}\right) \times\left(\mathrm{H}_{2} * \mathrm{~K}_{2}\right) \times \ldots \times\left(\mathrm{H}_{\mathrm{n}} * \mathrm{~K}_{\mathrm{n}}\right) \quad$ is nilpotent by Theorem (4). Therefore $\left(\mathrm{H}_{1} * \mathrm{~K}_{1}\right) \times\left(\mathrm{H}_{2} * \mathrm{~K}_{2}\right) \times \ldots \times\left(\mathrm{H}_{\mathrm{n}} * \mathrm{~K}_{\mathrm{n}}\right)$ is $\mathrm{M}$ group by Theorem (6). Hence $\left(\mathrm{H}_{1} * \mathrm{~K}_{1}\right) \times\left(\mathrm{H}_{2} * \mathrm{~K}_{2}\right) \times \ldots \times\left(\mathrm{H}_{\mathrm{n}} * \mathrm{~K}_{\mathrm{n}}\right) \quad$ is solvable by Theorem (5).

\section{Definition 8:}

Let $\mathrm{H}$ and $\mathrm{K}$ are two subgroups of the group $G$, then the derived subgroup or commutator subgroup of $\mathrm{G}$ denoted by $[\mathrm{H} * \mathrm{~K}, \mathrm{H} * \mathrm{~K}]$ where

$[\mathrm{H} * \mathrm{~K}, \mathrm{H} * \mathrm{~K}]=\left\{\prod\left[\mathrm{h}_{\mathrm{i}} * \mathrm{k}_{\mathrm{i}}, \mathrm{h}_{\mathrm{i}} * \mathrm{k}_{\mathrm{i}}\right], \mathrm{h}_{\mathrm{i}} * \mathrm{k}_{\mathrm{i}} \in\right.$ $\mathrm{H}_{\mathrm{i}} * \mathrm{~K}_{\mathrm{i}}, \mathrm{I}=1,2, \ldots, \mathrm{n}$, where $\Pi$ is a finite product with one or more factors $\}$.

\section{Theorem 9:}

The group $([\mathrm{H} * \mathrm{~K}, \mathrm{H} * \mathrm{~K}], *)$ is a normal subgroup of the group $(\mathrm{G}, *)$.

Proof:

Let $c$ an element of $[\mathrm{H} * \mathrm{~K}, \mathrm{H} * \mathrm{~K}]$ and $\mathrm{h} * \mathrm{k}$ is an element of $\mathrm{H} * \mathrm{~K}$. To prove that $(\mathrm{h} * \mathrm{k}) * \mathrm{c} *(\mathrm{~h} * \mathrm{k})^{-1} \in[\mathrm{H} * \mathrm{~K}, \mathrm{H} * \mathrm{~K}]$.

Now:

$(\mathrm{h} * \mathrm{k}) * \mathrm{c} *(\mathrm{~h} * \mathrm{k})^{-1}=\left[(\mathrm{h} * \mathrm{k}) * \mathrm{c} *(\mathrm{~h} * \mathrm{k})^{-1} * \mathrm{c}\right.$ $\left.\begin{array}{lll}- & 1\end{array}\right] * \mathrm{c}=[\mathrm{H} * \mathrm{~K}, \mathrm{c}] * \mathrm{c}$. Thus $(\mathrm{h} * \mathrm{k}) * \mathrm{c} *(\mathrm{~h} * \mathrm{k})^{-1} \in[\mathrm{H} * \mathrm{~K}, \mathrm{H} * \mathrm{~K}]$. Then $([\mathrm{H} * \mathrm{~K}, \mathrm{H} * \mathrm{~K}], *)$ is a normal subgroup of the group $(\mathrm{G}, *)$.

\section{Theorem 10:}

Let $\mathrm{G}^{\prime}$ be the normal subgroup of the group $\mathrm{H} * \mathrm{~K}$. Then $\left(\mathrm{H} * \mathrm{~K} / \mathrm{G}^{\prime}, \otimes\right)$ is abelian group iff $[\mathrm{H} * \mathrm{~K}, \mathrm{H} * \mathrm{~K}] \subseteq \mathrm{G}^{\prime}$.

Proof:

Let $\left(\mathrm{H} * \mathrm{~K} \quad / \mathrm{G}^{\prime}, \otimes\right)$ be an abelian group, thus for all $\left(\mathrm{h}_{1} * \mathrm{k}_{1}\right),\left(\mathrm{h}_{2} * \mathrm{k}_{2}\right)$ of $\mathrm{H} * \mathrm{~K}$

$\left(\mathrm{h} * \mathrm{k} * \mathrm{~h} \quad-\quad{ }^{1} * \mathrm{k}-{ }^{1}\right) * \mathrm{G}=$ $\left(\left(\mathrm{h}_{1} * \mathrm{k}_{1}\right) * \mathrm{G}^{\prime}\right) \otimes\left(\left(\mathrm{h}_{2} * \mathrm{k}_{2}\right) * \mathrm{G}^{\prime}\right) \otimes\left(\left(\mathrm{h}_{1} * \mathrm{k}_{1}\right)^{-}\right.$

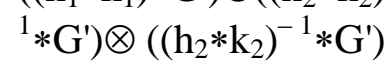

$=\left(\left(\mathrm{h}_{1} * \mathrm{k}_{1}\right) * \mathrm{G}^{\prime}\right) \otimes \quad\left(\left(\mathrm{h}_{1} * \mathrm{k}_{1}\right)^{-1} * \mathrm{G}^{\prime}\right) \quad \otimes$

$\left(\left(\mathrm{h}_{2} * \mathrm{k}_{2}\right) * \mathrm{G}^{\prime}\right) \otimes\left(\left(\mathrm{h}_{2} * \mathrm{k}_{2}\right)^{-1} * \mathrm{G}^{\prime}\right)$

$=\left(\left(\mathrm{h}_{1} * \mathrm{k}_{1}\right) *\left(\left(\mathrm{~h}_{1} * \mathrm{k}_{1}\right)^{-1} *\left(\mathrm{~h}_{2} * \mathrm{k}_{2}\right) *\left(\mathrm{~h}_{2} * \mathrm{k}_{2}\right)^{-}\right.\right.$

$\left.{ }^{1}\right) * \mathrm{G}^{\prime}$

$=\mathrm{e} * \mathrm{G}^{\prime}=\mathrm{G}^{\prime}$

Thus $[\mathrm{H} * \mathrm{~K}, \mathrm{H} * \mathrm{~K}] \subseteq \mathrm{G}^{\prime}$.

The convex

Let $[\mathrm{H} * \mathrm{~K}, \mathrm{H} * \mathrm{~K}] \subseteq \mathrm{G}^{\prime}$, for all $\left(\mathrm{h}_{1} * \mathrm{k}_{1}\right) * \mathrm{G}^{\prime}$, $\left(\mathrm{h}_{2} * \mathrm{k}_{2}\right) * \mathrm{G}^{\prime}$ of $\mathrm{H} * \mathrm{~K} / \mathrm{G}^{\prime}$. Thus

$\left(\left(\mathrm{h}_{1} * \mathrm{k}_{1}\right) *\left(\mathrm{~h}_{2} * \mathrm{k}_{2}\right)\right) * \mathrm{G}^{\prime} \otimes\left(\left(\mathrm{h}_{1} * \mathrm{k}_{1}\right) *\left(\mathrm{~h}_{2} * \mathrm{k}_{2}\right)\right)^{-}$

${ }^{1} * \mathrm{G}^{\prime}$

$=\left(\left(\mathrm{h}_{1} * \mathrm{k}_{1}\right) *\left(\mathrm{~h}_{2} * \mathrm{k}_{2}\right)\right) * \mathrm{G}^{\prime} \otimes \quad\left(\left(\mathrm{h}_{1} * \mathrm{k}_{1}\right)\right.$

$\left.{ }^{1} *\left(\mathrm{~h}_{2} * \mathrm{k}_{2}\right)^{-1}\right) * \mathrm{G}^{\prime}$

$=\left(\mathrm{h}_{1} * \mathrm{k}_{1}\right) *\left(\mathrm{~h}_{2} * \mathrm{k}_{2}\right) *\left(\mathrm{~h}_{1} * \mathrm{k}_{1}\right)^{-1} *\left(\mathrm{~h}_{2} * \mathrm{k}_{2}\right)^{-}$

${ }^{1} * \mathrm{G}^{\prime}$

$=\left(\mathrm{h}_{1} * \mathrm{k}_{1}\right) *\left(\mathrm{~h}_{2} * \mathrm{k}_{2}\right) *\left(\mathrm{~h}_{1} * \mathrm{k}_{1}\right)^{-1} *\left(\mathrm{~h}_{2} * \mathrm{k}_{2}\right)^{-1}$

$=\left[\left(\mathrm{h}_{1} * \mathrm{k}_{1}\right),\left(\mathrm{h}_{2} * \mathrm{k}_{2}\right)\right] \in \mathrm{G}^{\prime}$ 
$\left(\mathrm{h}_{1} * \mathrm{k}_{1}\right) *\left(\mathrm{~h}_{2} * \mathrm{k}_{2}\right) *\left(\mathrm{~h}_{1} * \mathrm{k}_{1}\right)^{-1} *\left(\mathrm{~h}_{2} * \mathrm{k}_{2}\right)^{-1} * \mathrm{G}^{\prime}$ $=\mathrm{G}^{\prime}$

$\left(\left(\mathrm{h}_{1} * \mathrm{k}_{1}\right) * \mathrm{G}^{\prime}\right) \otimes\left(\left(\mathrm{h}_{2} * \mathrm{k}_{2}\right) * \mathrm{G}^{\prime}\right)=\mathrm{G}^{\prime}$

Thus

$\left(\left(\mathrm{h}_{1} * \mathrm{k}_{1}\right) *\left(\mathrm{~h}_{2} * \mathrm{k}_{2}\right)\right) * \mathrm{G}^{\prime}=\left(\left(\mathrm{h}_{2} * \mathrm{k}_{2}\right) *\left(\mathrm{~h}_{1} * \mathrm{k}_{1}\right)\right) * \mathrm{G}^{\prime}$

$\left(\left(\mathrm{h}_{1} * \mathrm{k}_{1}\right) * \mathrm{G}^{\prime}\right) \otimes\left(\left(\mathrm{h}_{2} * \mathrm{k}_{2}\right) * \mathrm{G}^{\prime}\right)=\left(\left(\mathrm{h}_{2} * \mathrm{k}_{2}\right) * \mathrm{G}^{\prime}\right) \otimes((\mathrm{h}$ $\left.\left.{ }_{1} * \mathrm{k}_{1}\right) * \mathrm{G}^{\prime}\right)\left(\mathrm{H} * \mathrm{~K} / \mathrm{G}^{\prime}, \otimes\right)$ is abelian group

\section{Theorem 11:}

Let $(\mathrm{H} * \mathrm{~K})^{\prime}$ be the normal subgroup of the group $\mathrm{H} * \mathrm{~K}$. Then $(\mathrm{H} * \mathrm{~K}$ $\left./(\mathrm{H} * \mathrm{~K})^{\prime}, \otimes\right)$ is an M-group if and only if $[\mathrm{H} * \mathrm{~K}, \mathrm{H} * \mathrm{~K}] \subseteq(\mathrm{H} * \mathrm{~K})^{\prime}$.

\section{Proof:}

(1) Suppose that $(\mathrm{H} * \mathrm{~K}) /(\mathrm{H} * \mathrm{~K})^{\prime}$ is an $\mathrm{M}$ group to prove $[\mathrm{H} * \mathrm{~K}, \mathrm{H} * \mathrm{~K}] \subseteq(\mathrm{H} * \mathrm{~K})^{\prime}$.

Let $\mathrm{a}, \mathrm{b} \in \mathrm{H} * \mathrm{~K}$, to show $[\mathrm{a}, \mathrm{b}] \in(\mathrm{H} * \mathrm{~K})^{\prime}$ since $a, b \in H * K$ so $a(H * K)^{\prime}, b(H * K)^{\prime}$ $\in \mathrm{H} * \mathrm{~K} /(\mathrm{H} * \mathrm{~K})^{\prime}$

$\left(\mathrm{a}(\mathrm{H} * \mathrm{~K})^{\prime}\right)\left(\mathrm{b}(\mathrm{H} * \mathrm{~K})^{\prime}\right)=\left(\mathrm{b}(\mathrm{H} * \mathrm{~K})^{\prime}\right)\left(\mathrm{a}(\mathrm{H} * \mathrm{~K})^{\prime}\right)$

(ab) $(\mathrm{H} * \mathrm{~K})^{\prime}=(\mathrm{ba})(\mathrm{H} * \mathrm{~K})^{\prime} \mathrm{aba}^{-1} \mathrm{~b}^{-1} \in$ $(\mathrm{H} * \mathrm{~K})^{\prime}\left((\mathrm{H} * \mathrm{~K})^{\prime}\right.$ is normal subgroup) $[a, b] \in(\mathrm{H} * \mathrm{~K})^{\prime}$, where $\mathrm{a}=\mathrm{h}_{1} * \mathrm{k}_{1}, \mathrm{~b}=$ $\mathrm{h}_{1} * \mathrm{k}_{1}, \mathrm{~h}_{1}, \mathrm{~h}_{2} \in \mathrm{H}, \mathrm{k}_{1}, \mathrm{k}_{2} \in \mathrm{K}$.

Thus $[\mathrm{H} * \mathrm{~K}, \mathrm{H} * \mathrm{~K}] \subseteq(\mathrm{H} * \mathrm{~K})^{\prime}$.

(2) Suppose that $[\mathrm{H} * \mathrm{~K}, \mathrm{H} * \mathrm{~K}] \subseteq(\mathrm{H} * \mathrm{~K})^{\prime}$ $[a, b] \in(H * K)^{\prime}, \forall a, b \in \mathrm{H} * \mathrm{~K}$ $\mathrm{aba}^{-1} \mathrm{~b}^{-1} \in(\mathrm{H} * \mathrm{~K})^{\prime}$

$(\mathrm{a}, \mathrm{b})(\mathrm{ba})^{-1} \in(\mathrm{H} * \mathrm{~K})^{\prime}$

$(\mathrm{a}, \mathrm{b})(\mathrm{H} * \mathrm{~K})^{\prime}=(\mathrm{ba})(\mathrm{H} * \mathrm{~K})^{\prime}$

$(\mathrm{H} * \mathrm{~K}) /(\mathrm{H} * \mathrm{~K})^{\prime}$ is commutative, by Theorem (8)

$(\mathrm{H} * \mathrm{~K}) /(\mathrm{H} * \mathrm{~K})^{\prime}$ is nilpotent by

Theorem (4).

$(\mathrm{H} * \mathrm{~K}) /(\mathrm{H} * \mathrm{~K})^{\prime} \quad$ is M-group by Theorem (6).

\section{Corollary 12:}

Let $S_{n}$ be a symmetric group and $\mathrm{H}_{1}, \mathrm{H}_{2}, \ldots, \mathrm{H}_{\mathrm{n}}$ are normal subgroup of $\mathrm{S}_{\mathrm{n}}$. Then $\mathrm{S}_{\mathrm{n}} /\left(\mathrm{H}_{1} \cap \mathrm{H}_{2} \cap \ldots \cap \mathrm{H}_{\mathrm{n}}\right)$ is Mgroup.

\section{Proof: Clear.}

\section{Example 13:}

$\mathrm{S}_{4}$ is the symmetric group of order 24 , the subgroup

$\mathrm{H}_{32}=\mathrm{A}_{4}=\{\mathrm{i},(123),(132),(124),(134)$, (234), (243), (142), (143), (12) (34), (13) (24), (14) (23)\} of order 12 is a normal subgroup of $\mathrm{S}_{4}$, thus $\mathrm{S}_{4} / \mathrm{A}_{4}$ is an $\mathrm{M}$ group.

\section{Example 14:}

Let GL $(4, R)$ be the general linear group of non-singular $4 \times 4$ matrices over $\mathrm{R}$ and let $\mathrm{SL}(4, \mathrm{R})$ be the special linear group of $4 \times 4$ matrices each having determinant 1 . Then GL $(4, \mathrm{R}) / \mathrm{SL}(4, \mathrm{R})$ is an M-group, hence it is a solvable group.

\section{Solution:}

In the beginning we prove that $\mathrm{SL}(4, \mathrm{R})$ is the derived group of $\mathrm{GL}(4, \mathrm{R})$. We use the result that if $x, y$ are $n \times n$ matrices then $\operatorname{det}(\mathrm{xy})=\operatorname{det}(\mathrm{x}) \operatorname{det}(\mathrm{y})$.

This relation asserts that mapping $\mathrm{x} \longrightarrow \operatorname{det}(\mathrm{x}) \quad(\mathrm{x} \in \mathrm{GL}(4, \mathrm{R})) \quad$ is $\quad \mathrm{a}$ homomorphism of GL $(4, R)$ into $R^{*}$, the multiplicative group of non-zero real numbers.

The kernel of this homomorphism is precisely SL $(4, R)$ which is a normal subgroup of GL(4,R).

Furthermore, since $\mathrm{R}^{*}$ is abelian [GL $(4, \mathrm{R})]^{\prime} \subseteq \mathrm{SL}(4, \mathrm{R})$. The difficult part of our argument is to show that $\operatorname{SL}(4, \mathrm{R}) \subseteq$ $[\mathrm{GL}(4, \mathrm{R})]^{\prime}$ or equivalently, to show that every $4 \times 4$ matrices of determinant 1 is a product of commutators, we consider some simple matrices of determinant 1 namely:

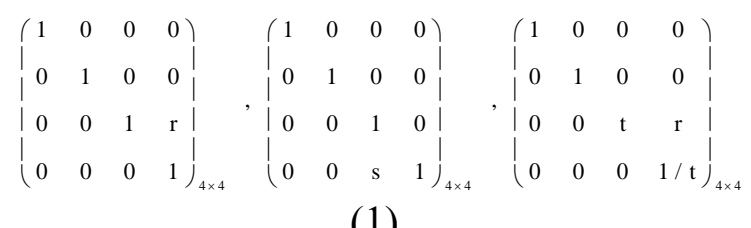

(where $\mathrm{r}, \mathrm{s}, \mathrm{t} \in \mathrm{R}, \mathrm{t} \neq 0$ ).

The following matrix calculation, then, shows that these matrices are acctually commutators: 


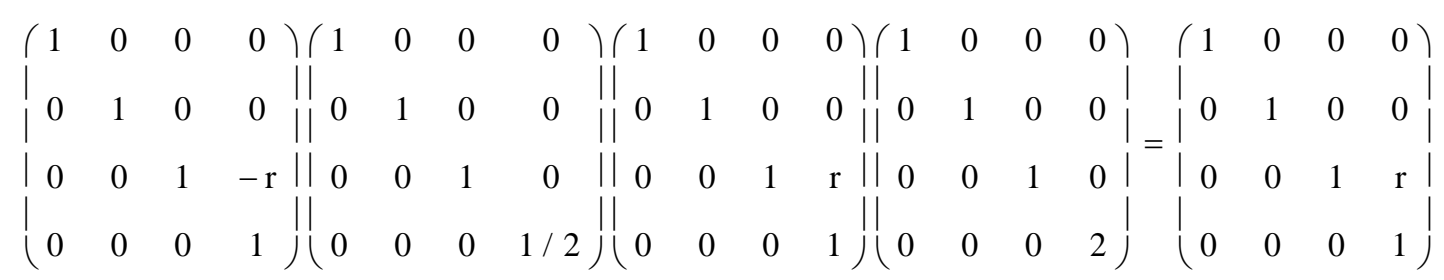

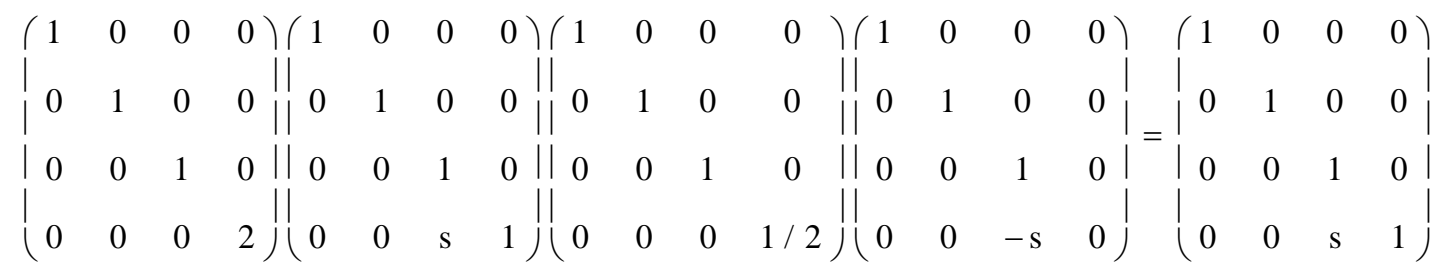

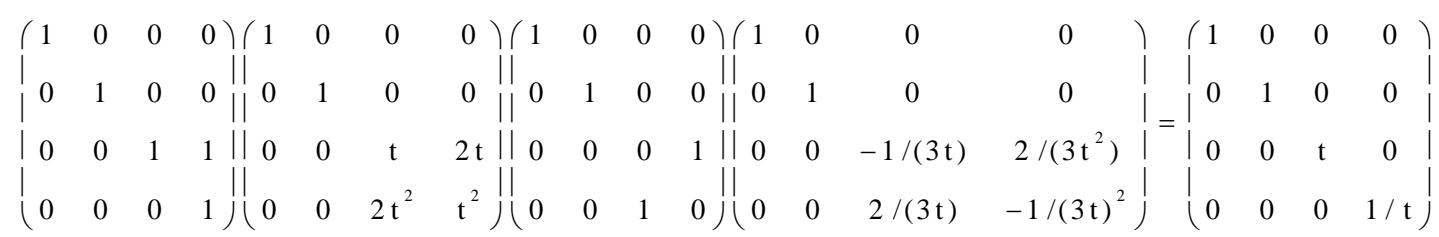

The matrices of (1) also generate GL(4,R) since if $\left\{\begin{array}{llll}1 & 0 & 0 & 0 \\ 0 & 1 & 0 & 0 \\ 0 & 0 & \mathrm{a} & \mathrm{b} \\ 0 & 0 & \mathrm{c} & \mathrm{d}\end{array}\right) \in \mathrm{SL}(4, \mathrm{R})$ then

$\mathrm{a}, \mathrm{d}, \mathrm{b}, \mathrm{c} \in \mathrm{R}$ and $\mathrm{ad}-\mathrm{bc}=1$ and if $\mathrm{c} \neq 0$ we have

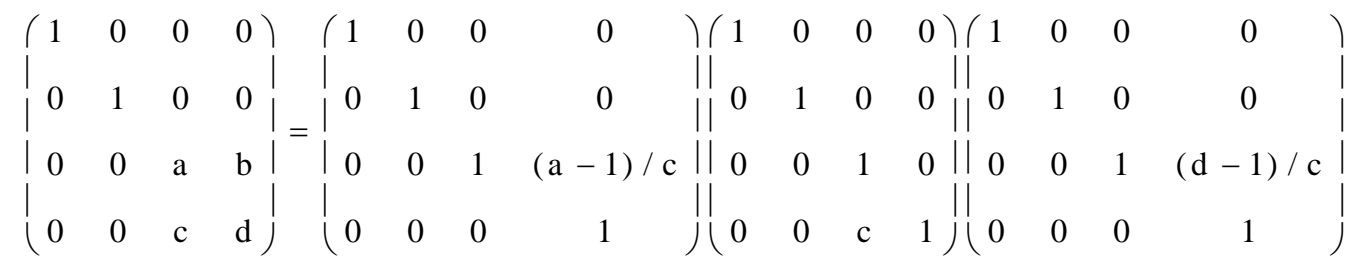

And if $\mathrm{c}=0$ we have

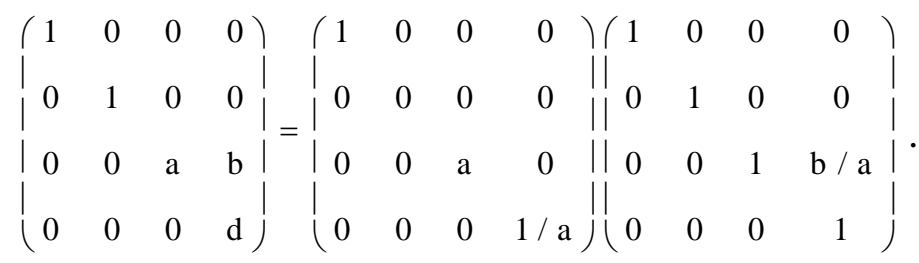

Thus SL $(4, R)$ is generated by commentators and so $\mathrm{SL}(4, \mathrm{R}) \subseteq$ $[\mathrm{GL}(4, \mathrm{R})]^{\prime}$. Then SL $(4, \mathrm{R})$ is the derived group of $\mathrm{GL}(4, \mathrm{r})$. Now GL $(4, \mathrm{R})$ / $\mathrm{SL}(4, \mathrm{R})$ is M-group by Theorem (6) and solvable by Theorem (5)

\section{References:}

[1]Borel, A. 1991. Algebraic Linear Groups, Springer-Verlag, New York, USA. pp.288.
[2]Rotman, J. 1995. An Introduction to the Theory of Groups, SpringerVerlag, New York, USA.pp.513.

[3]Oystein, O. 2014. Theory of Monomial Groups, Texts and Readings in Mathematics/ 46, paper cover, Rs.415.00, Hindustan Book Agency, Privcy Policy\ Contact US, 236 pages.

[4]Ralph, B. C. 2015. Monomial Groups, Texts and Readings in Mathematics/ 47, paper cover, 
Rs.416.00, Hindustan Book Agency, Privcy Policyl Contact US, 250 pages.

[5]Lakshmibai, V. 2011. The development of standard monomial theory. II, A tribute to C. S. Seshadri (Chennai, 2002), Trends Math., Basel, Boston, Berlin: Birkhäuser, pp. 283-309, ISBN 9783-7643-0444-7, MR 2017589.

[6]Musili, C. 2011. The development of standard monomial theory. I, A tribute to C. S. Seshadri (Chennai, 2002), Trends Math., Basel, Boston, Berlin: Birkhäuser, pp. 385420, ISBN 978-3-7643-04447, MR 2017594.

[7]Seshadri, C. S. 2012. Standard monomial theory-a historical account", Collected papers of C. S. Seshadri. Volume 2. Schubert geometry and representation theory., New Delhi: Hindustan Book Agency, pp. 3-50, ISBN 9789380250175 , MR 290589.

[8]Bravi P., Chirivi R., Gendini J. and Maffei A. 2015. Standard Monomial Theory for Woderful Varieties, arXiv:1504.02387v1 [math.AG] 9Apr 2015.

[9]Lang, S. 2002. Algebra, SpringerVerlag, New York, USA. pp.914.

[10] Navarro, G. 1995. Primitive Characters of Subgroups of Monomial Groups, Math. Z 128: 439445.

[11] Hajim, A. A. 2007. On Representations of Monomial Groups, M.Sc. Thesis, College of Science, Al-Mustansiriya University, Iraq, pp.63-65.

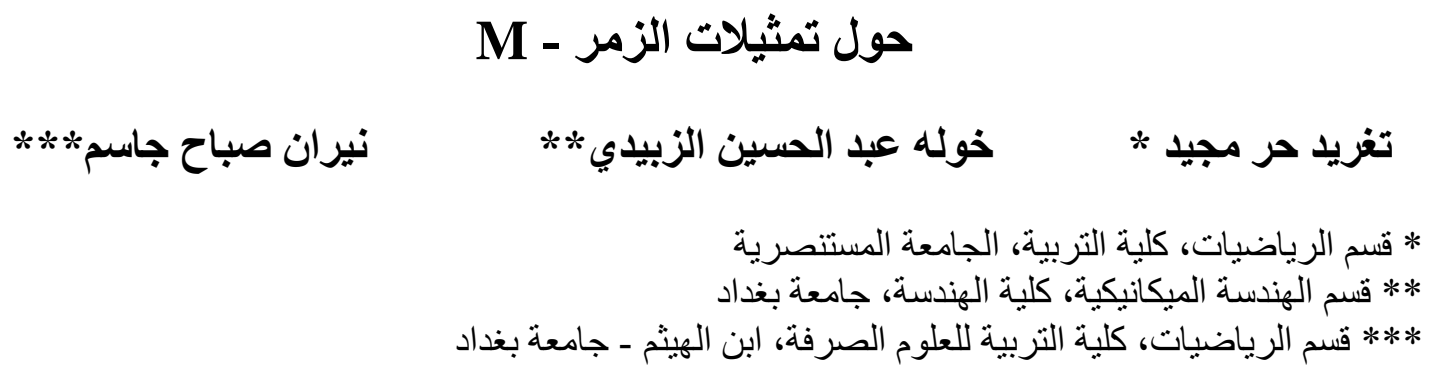

\footnotetext{
الخلاصة:

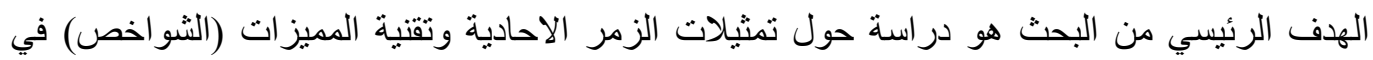

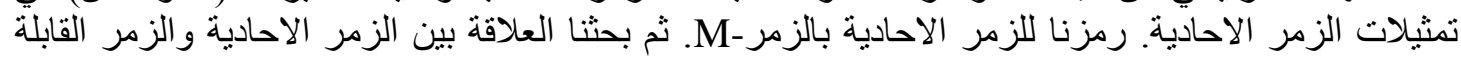

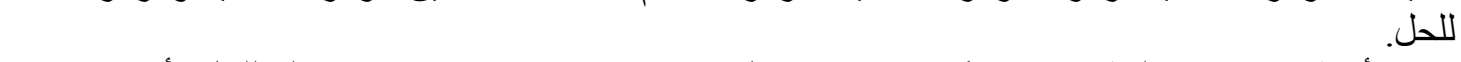

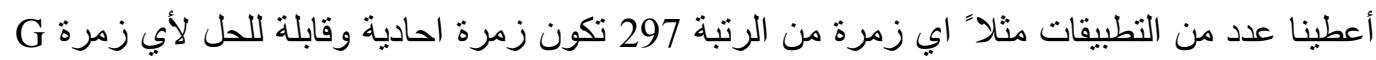

تكون زمرة القسمة 'G/G

الكلمات المفتاحية: نظرية التمثيل، زمرة أحادية، زمرة قابلة للحل، تمثيل الزمرة الاحادية.
} 